\title{
Die Stapesplastik aus pflegerischer Sicht im HNO-OP
}

\author{
Anja Ackermann
}

\section{Zusammenfassung}

Da es in der Literatur, wie zum Beispiel dem Pflegeleitfaden OP, sehr wenig über die Hals-Nasen-Ohren-Heilkunde zu erfahren gibt und nur knapp über Standardoperationen berichtet wird, habe ich mich dazu entschlossen, etwas über die Stapesplastik zu schreiben. Sie ist eine Operation, die hohe Anforderungen an das OP-Personal stellt. Ein überdurchschnittliches Maß an Disziplin, Ruhe, Geduld, Fingerspitzengefühl und vor allem Konzentration ist notwendig, um diesen anspruchsvollen Eingriff kompetent $\mathrm{zu}$ meistern. Daneben fordert der Umgang mit den schwerhörigen Patienten eine ausgeprägte Empathie für die speziellen Bedürfnisse dieser Betroffenen.

\section{Stapedioplasty in ENT Operations from the Nursing Point of View}

Since very little information is given in the literature, for example in the operative care guidelines, on otorhinolaryngeal care and the standard operations are only briefly described, I have decided to present a report on stapedioplasty. This is an operation that places high demands on the operating room personnel. Above average degrees of discipline, calmness, patience, tact and, above all, concentration are necessary in order to perform this operation competently. In addition, interaction with the hearing impaired patient demands a pronounced empathy for the special requirements of these patients.

\section{Krankheitsbild}

Die Otosklerose ist eine Erkrankung, welche durch Umbau der knöchernen Labyrinthkapsel des Innenohrs gekennzeichnet ist. Die Genese ist bislang ungeklärt. Sie tritt häufiger bei Frauen als Männern auf und manifestiert sich meist zwischen dem 20. und 40. Lebensjahr. Ein Symptom der Otosklerose kann neben der subjektiv empfundenen Hörminderung auch ein Ohrgeräusch (Tinnitus) auf der betroffenen Seite sein [3].

\section{Lokalbefund}

Bei der ohrmikroskopischen Untersuchung zeigt sich ein regelrechter Trommelfellbefund, die Tube (Ohrtrompete) ist frei durchgängig.

OP-JOURNAL 2009; 25: 64-66

(c) Georg Thieme Verlag KG Stuttgart · New York DOI 10.1055/s-0029-1185494

In seltenen Fällen scheint das Promontorium wegen der vermehrten Gefäßzeichnung rot durch das Trommelfell hindurch [4].

\section{Diagnostik}

Die Diagnose wird durch den Hals-Nasen-Ohren-Arzt gestellt. Aufgrund des meist unauffälligen Ohrbefunds nimmt die audiometrische Diagnostik einen besonderen Stellenwert ein.

In der Reintonaudiometrie zeigt sich eine Schallleitungsschwerhörigkeit. Wegen der Fixation des Steigbügels kann der Schall nicht verlustfrei vom Trommelfell auf das Innenohr übertragen werden, daher fehlen auch die Stapediusreflexe [2].

\section{Therapie}

Konservativ ist eine Otosklerose kaum zu beeinflussen. Eine Hörgeräteversorgung ist möglich, wird von den Patienten jedoch meist nicht gewünscht. Daher stellt die Stapesplastik eine mögliche Alterna- tive zur Wiederherstellung des durch die Stapesfixation behinderten Gehörs dar.

$\mathrm{Zu}$ unterscheiden sind 2 Arten der Operationsdurchführung:

\section{Stapesplastik in Vollnarkose}

Der Patient wird auf dem Rücken gelagert. Ein fakultatives intraoperatives Monitoring des $\mathrm{N}$. facialis wird angeschlossen. Der Inzisionsbereich wird mit einem Lokalanästhetikum (mit Adrenalinzusatz) infiltriert.

Nach sterilem Abdecken des OP-Feldes, Anschließen des OP-Saugers und der bipolaren Pinzette sowie der korrekten Positionierung des OP-Mikroskops durch die OP-Pflegekraft kann die Operation beginnen.

Unter Zuhilfenahme eines Nasenspekulums wird der Gehörgangseingang gespreitzt. Mit einem 10er-Skalpell wird in der Mitte des knöchernen Gehörgangs, unter Knochenkontakt beginnend, ein Schnitt nach außen geführt. Dieser wird mit einem Sperrer offengehalten. Je nach Einblick ist ein weiterer Sperrer im rechten Winkel einzusetzen. Etwa $5 \mathrm{~mm}$ vom Trommelfell entfernt durchtrennt man mit einem geraden Rundmesser die hintere Gehörgangshaut bis auf den Knochen. Unter festem Knochenkontakt wird der Anulus des Trommelfells unter Schonung der Chorda tympani(Schmecknerv) ausgelöst und der sog. tympanomeatale Lappen vorgeschoben.

Mit Hilfe des House-Löffels wird der Knochen der Gehörgangshinterwand abgetragen, damit die Sicht auf die Gehörknöchelchenkette ermöglicht wird. Durch vorsichtiges Berühren an den Gehörknöchelchen wird die Beweglichkeit von Hammer und Amboss getestet. Typischerweise sind Hammer und Amboss mobil, während der Steigbügel fixiert ist (Abb. 4). Die Distanz zwischen Fußplatte und Amboss misst man mit einem auf 


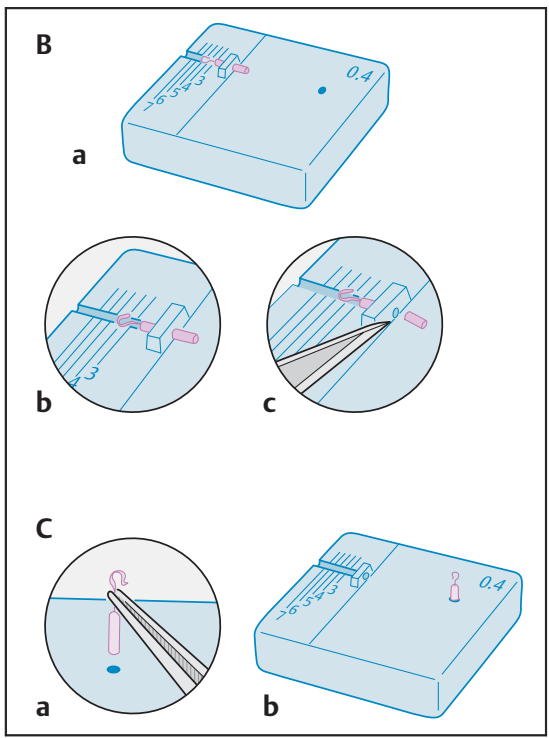

Abb. 1 Zuschneiden der Prothese und Einsetzen der Stapesprothese in den Stapesblock.

die Fußplatte aufgesetzten Längenmesser nach Fisch anhand der Markierung. Danach wird die Prothesenlänge an die gemessene Distanz angepasst, sie sollte jedoch 0,3 mm länger sein (Abb.1). Mit einem $90^{\circ}$-Häkchen trennt man das Amboss-Steigbügel-Gelenk auf, im Anschluss wird die Stapediussehne mit einem Sichelmesser gekappt.

Um den hinteren Schenkel des Stapes möglichst fußplattennah zu durchtrennen, wird häufig ein feiner Bohrer (Skeeter) verwendet. Jetzt kommen die 4 verschiedenen Perforatoren nach Fisch zum Einsatz, diese reichen von $0,3 \mathrm{~mm}$ bis 0,6 mm Durchmesser (Abb. 2); mit dem kleinsten wird begonnen. Hierbei ist äußerste Vorsicht geboten, damit die Fußplatte oder Fragmente davon nicht ins Vestibulum des Innenohrs absinken und permanenten Schwindel verursachen.

Der Durchmesser der Öffnung wird anschließend mit einem Messzylinder (Abb.3) überprüft.

\section{Im Idealfall beträgt sie $0,6 \mathrm{~mm}$.}

Die zurechtgeschnittene Prothese wird nun mit Hilfe des Ohrzängelchens nach Hartmann zunächst über den Amboss eingehängt und dann vorsichtig in die Perforation der Fußplatte eingeschoben. Mit Hilfe der Crimpzange wird die Prothese um den langen Ambossschenkel geschlossen.

Kleine Bindegewebsstückchen werden um die Prothese gelegt, zum Abdichten

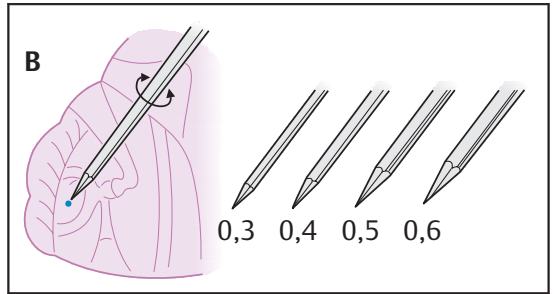

Abb. 2 Perforatoren in verschiedenen Größen.

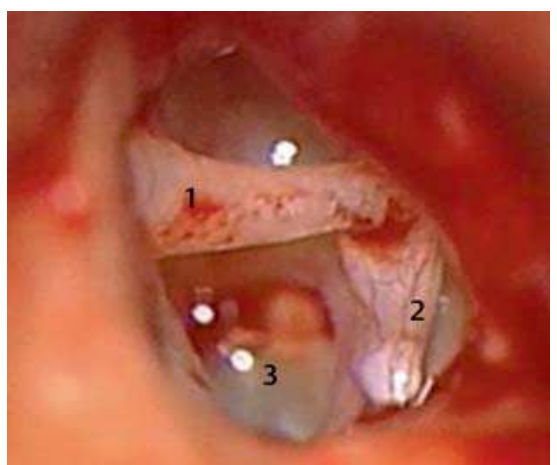

Abb. 4 Situs im Mittelohr: Amboss (1), Steigbügelköpfchen (2), Fußplatte (3).

der Fußplattenregion. Man verhindert somit eine Perilymphfistel (Abb. 5).

Der Gehörgangslappen wird zurückverlegt und 2-3 Silikonschienen werden positioniert, um den Lappen in gewünschter Position zu halten.

Die Folien wiederum werden mit einer Vaselinsalbenstreifentamponade fixiert. Der Sperrer wird entnommen und das Wundgebiet mit einer 5/0-Hautnaht verschlossen. Abschließend wird ein zirkulärer, leicht komprimierender Ohrverband angelegt und die Operation beendet.

\section{Stapesplastik in Lokalanästhesie}

Das operative Vorgehen in Lokalanästhesie gleicht dem in Vollnarkose. Da der Patient wach ist, kann man durch Zuhalten des anderen Ohres einen vereinfachten Hörtest durchführen. Der Operateur nennt in Flüstersprache einige Zahlen, die der Patient wiederholen soll. Somit kann man noch während der Operation die Hörverbesserung prüfen und ggf. Korrekturen an der Prothesenlage bzw. -länge durchführen. Bei zu tiefem Eintauchen des Implantats ins Vestibulum kann sehr heftiger Schwindel auftreten. In seltenen Fällen kann auch mit Übelkeit beim Patienten gerechnet werden. Meistens sind diese Beschwerden nur vorübergehend.

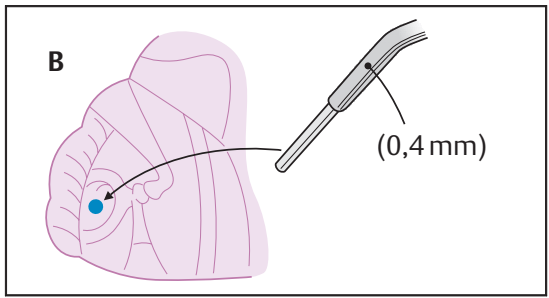

Abb. 3 Messzylinder.

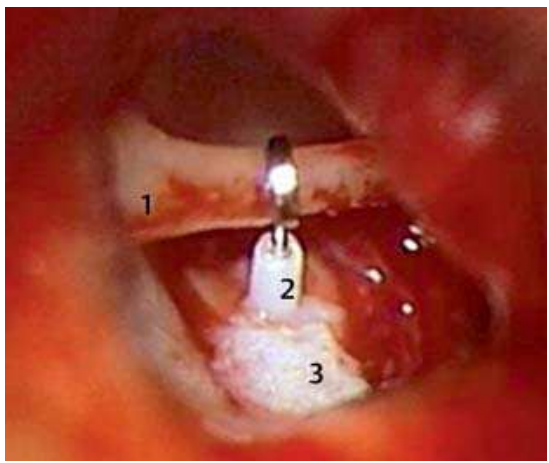

Abb. 5 Platin-Teflon-Prothese (2) am langen Ambossschenkel (1) mit Bindegewebsstückchen der ovalen Nische (3).

\section{Warum ist diese Operation so besonders?}

Es handelt sich um einen Routineeingriff und eigentlich geht es „nur“ um das Ohr. Allerdings wird eine Schwerhörigkeit von gut hörenden Menschen im Allgemeinen stark unterschätzt. Hören ist der soziale Sinn des Menschen! Schwerhörige sind häufig besonders ängstlich und auch misstrauisch, da sie in ihrer Kommunikation beeinträchtigt sind. Dies gilt vor allem im Operationsbetrieb, wo sie wegen der Gesichtsmasken nicht von den Lippen unterstützend ablesen können.

Zum anderen liegt bei kaum einer Operation der Erfolg so nahe an der Katastrophe. Die kleinen Strukturen und die Notwendigkeit, die Innenohrräume eröffnen zu müssen, beinhalten das stete Risiko einer Ertaubung oder des Auftretens invalidisierenden Schwindels. Bei keinem Engriff geht es daher derart konzentriert, ruhig und gleichzeitig angespannt zu wie bei der Stapesplastik. Das betrifft nicht nur den Operateur, sondern bedeutet auch für die OP-Pflegekraft höchste Aufmerksamkeit. Wünschenswert ist eine exakte Kenntnis der einzelnen Operationsschritte, um die nächste Handlung antizipieren und die entsprechenden Instrumente reichen zu können. Da diese mit bloßem Auge z.T. nicht zu unterscheiden sind, bedarf es großer Umsicht 
des OP-Personals am Tisch, um diese Schwierigkeiten souverän zu meistern. Im optimalen Fall bekommt der Operateur diese Anspannung der instrumentierenden Person nicht mit und kann sich voll seinen eigenen Aufgaben widmen.

Diese Mischung aus Anspannung und Konzentration, kombiniert mit einem winzigen, delikaten OP-Gebiet sowie evtl. unberechenbare Reaktionen des Patienten bei Eingriff in Lokalanästhesie machen das Besondere dieser „Königsoperation“ aus, deren gekonnte Assistenz von dem wiedererlangten Hören des Patienten unmittelbar belohnt wird.

\section{Ausbildung}

Neben der perfekten Assistenz stellt sich für Personen in Leitungsfunktionen die verantwortungsvolle Aufgabe, derartig delikate Tätigkeiten an die unterstellten Mitarbeiter weiterzugeben. Ein Problem liegt unter anderem darin, dass sich so etwas nur bedingt „üben“ lässt. Voraussetzung ist ein einfühlsames Heranführen über Vermittlung theoretischer Kenntnisse und praktischer Fähigkeiten bei anderen, weniger diffizilen mikrochirurgischen Eingriffen. Nur so lässt sich eine vertretbare Qualität der OP-Assistenz schon zu Beginn ohne vermeidbare Frustration des Mitarbeiters erreichen, was der ausbildenden Person ein überdurchschnittliches Ausmaß an fachlicher und sozialer Kompetenz abverlangt.

\section{Literatur}

1 Debrand-Passard A, Wunderle G. Pflegeleitfaden OP. Lübeck, Stuttgart, Jena, Ulm: Urban und Fischer bei Elsevier; 1996; 64: 155-512

2 Fisch $U$. Tympanoplasty, mastoidectomy and stapes surgery. Stuttgart: Thieme; 1994

3 Probst R, Grevers G, Iro H. Hals-Nasen-OhrenHeilkunde. Stuttgart: Thieme; 2008

${ }^{4}$ Theissing J. HNO-Operationslehre. 3. Aufl. Stuttgart: Thieme; 1996: 378-385

${ }^{5}$ Helms J, Jahrdoerfer RA. Kopf- und Hals-Chirurgie, Band 2. Stuttgart: Thieme; 1996: $230-262$

\section{Anja Ackermann}

Abteilungsleitung HNO-OP/Ambulanz

Klinik für Hals-Nasen-

Ohrenheilkunde, Kopf-, Hals- und plastische Gesichtschirurgie

Klinikum Bad Hersfeld

Seilerweg 29

36251 Bad Hersfeld

anja.ackermann@klinikum-hef.de 